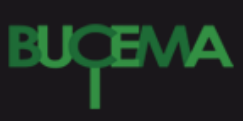

Bulletin du centre d'études médiévales d'Auxerre | BUCEMA

Hors-série $n^{\circ} 10 \mid 2016$

L'origine des sites monastiques : confrontation entre la terminologie des sources textuelles et les données archéologiques

\title{
L'origine des sites monastiques. Confrontation entre la terminologie des sources textuelles et les données archéologiques
}

Sébastien Bully

\section{(2) OpenEdition \\ Journals}

\section{Édition électronique}

URL : https://journals.openedition.org/cem/14465

DOI : $10.4000 /$ cem. 14465

ISSN : 1954-3093

Éditeur

Centre d'études médiévales Saint-Germain d'Auxerre

\section{Référence électronique}

Sébastien Bully, «L'origine des sites monastiques. Confrontation entre la terminologie des sources textuelles et les données archéologiques », Bulletin du centre d'études médiévales d'Auxerre | BUCEMA [En ligne], Hors-série $n^{\circ} 10$ | 2016, mis en ligne le 09 décembre 2016, consulté le 04 mars 2023. URL : http://journals.openedition.org/cem/14465; DOI : https://doi.org/10.4000/cem.14465

Ce document a été généré automatiquement le 4 mars 2023.

\section{cc) (1) ()}

Creative Commons - Attribution - Pas d'Utilisation Commerciale - Partage dans les Mêmes Conditions 4.0 International - CC BY-NC-SA 4.0

https://creativecommons.org/licenses/by-nc-sa/4.0/ 


\title{
L'origine des sites monastiques. Confrontation entre la terminologie des sources textuelles et les données archéologiques
}

\author{
Sébastien Bully
}

En guise d'introduction...

1 Pour ces quatrièmes Journées d'études monastiques ${ }^{1}$, nous avons souhaité aborder la question de l'origine des sites monastiques à partir d'une confrontation entre la terminologie des sources textuelles et les données archéologiques. À l'instar des rencontres précédentes, il s'agit d'engager une discussion entre archéologues et historiens des sources écrites, afin, ici, de réfléchir ensemble sur les définitions archéologiques, monumentales, architecturales ou topographiques que recouvrent les différents termes utilisés dans la documentation textuelle pour des périodes - entre l'Antiquité tardive et le haut Moyen Âge - et/ou des contextes différents de fondations. Sans bien évidemment prétendre à l'exhaustivité, c'est à partir d'un panel de nouveaux programmes de recherches représentatifs, historiques aussi bien qu'archéologiques, en France comme à l'étranger, que nous avons élaboré cette rencontre.

2 Cette réflexion doit permettre de formaliser de (souvent) longues discussions engagées entre nous sur différents chantiers et de soumettre à la communauté de nouvelles hypothèses ou, parfois, de simples pressentiments attendant encore d'être nourris...

3 La démarche se veut complémentaire de celles engagées, par exemple, par Brigitte Beaujard dans son étude sur le culte des saints en Gaule : selon cette dernière le terme de cella pouvait en effet définir un monument abritant une tombe sainte, et pas seulement un modeste établissement ${ }^{2}$. Ces aspects sémantiques ont été également déjà bien traités pour l'Italie par Gisella Cantino Wataghin, notamment pour le terme de monasterium, dont elle a démontré toute la polysémie et qui peut indiquer à la fois une communauté ou un établissement d'ermite(s) ou, encore, comme dans les sources ravennates jusqu'au IX ${ }^{e}$ siècle, une simple chapelle ou un oratoire ${ }^{3} . .$. 
4 On sait bien toutes les interrogations que suscitent les villae du haut Moyen Âge mentionnées dans les chartes de fondations ou des Vitae précoces, et dans lesquelles s'installèrent un certain nombre de nos monastères, entre domaine foncier et structures résidentielles. On pensera à la fondation de Fulda qui réutilise au milieu du $\mathrm{VIII}^{\mathrm{e}}$ siècle les structures d'une villa rustica $\mathrm{du}_{\mathrm{VII}}{ }^{\mathrm{e}}$ siècle ${ }^{4}$. Les dernières recherches menées par Christian Sapin et Anne Baud à Cluny sont d'ores et déjà des plus éclairantes, comme c'est le cas également des travaux de Pascale Chevalier, Arlette Maquet et Sophie Liégard sur la villa de Sylviniacum, qui deviendra le prieuré de Souvigny. C'est malheureusement moins vrai pour la villa de Gigny, domaine de Bernon, dont on ignore encore tout. Et qu'en est-il de ces fondations de l'Antiquité tardive qui s'installent dans des villae antiques, comme à Ligugé où à Vivarium, pour n'en citer que deux parmi les plus célèbres, ou de celles du haut Moyen Âge, comme la fondation primitive de Vézelay dans la villa supposée de Saint-Père? Loin d'être un "marronnier» de l'historiographie monastique, la question de réutilisation des structures de l'Antiquité par les premiers monastères demeure complexe, riche et surtout peu étudiée pour la Gaule - contrairement à l'Espagne par exemple ${ }^{5}$ et à l'Italie dans une moindre mesure ${ }^{6}$. À cet égard, on pensera aux interrogations que suscite l'interprétation de la description de la villa-monastère de Primuliacum de Sulpice Sévère $e^{7}$, qui nous renvoie, finalement, à la question : qu'est-ce qu'un monastère à la fin $\mathrm{du} \mathrm{IV}^{\mathrm{e}}-\mathrm{v}^{\mathrm{e}}$ siècle ? À n'en pas douter, pour la région, des travaux sur les conditions de fondation de Condat/Saint-Claude au v $v^{e}$ siècle pourraient nous amener sur ce terrain.

5 Enfin, j'achèverai cette rapide introduction en m'arrêtant au monastère dans lequel nous nous sommes retrouvés, Balma, qui est cité pour la première fois dans un acte de 869 comme une cellula, en opposition ou en complément, dans ce même document, à l'abbatiolia de Château-Chalon. Dans sa confrontation avec les données archéologiques issues des dernières recherches menées sur Baume, la définition de cellula, entendue comme une simple station foncière, doit être fortement nuancée. Tout cela nous ramène à l'interrogation posée par Gérard Moyse il y a quelques années - et à laquelle nous allons essayer de répondre en partie - lorsqu'il écrivait à propos de Baume: " quand saurons-nous exactement le sens d'un mot comme cellula au haut Moyen Âge? ${ }^{8} »$. 
Fig. 1 - Communication dans la « salle de l'ancien dortoir des moines » à Baume-les-Messieurs le 5 septembre 2014

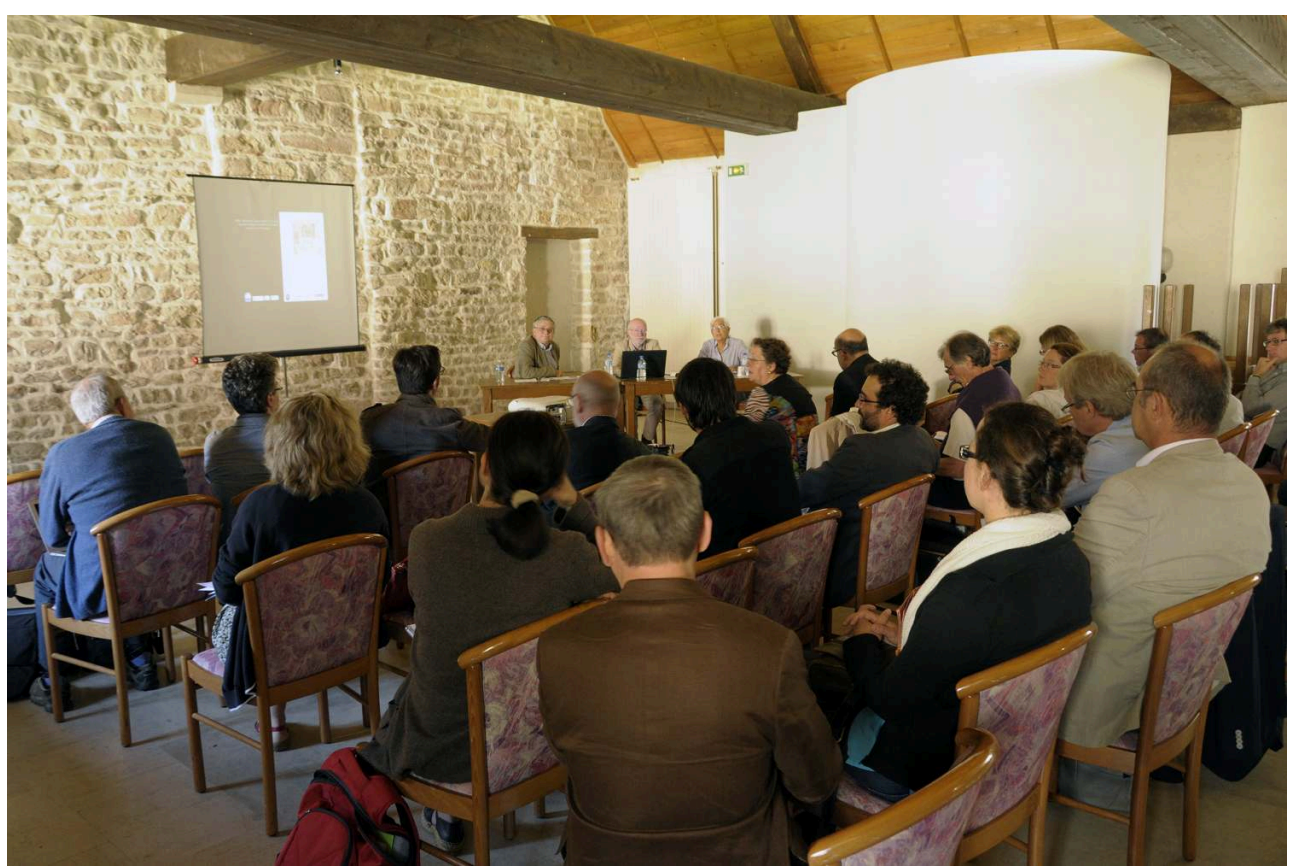

Cl. D. Vuillermoz

\section{NOTES}

1. Comme pour les années précédentes, ces journées d'études ont été organisées dans le cadre du Projet collectif de recherche intitulé « Monastères en Europe occidentale ( $\mathrm{v}^{\mathrm{e}}-\mathrm{x}^{\mathrm{e}}$ siècle). Topographie et structures des premiers établissements en Franche-Comté et en Bourgogne", sous la codirection de S. Bully et $\mathrm{C}$. Sapin.

2. B. BEAUJARD, Le culte des saints en Gaule. Les premiers temps. D'Hilaire de Potiers à la fin du $\mathrm{VI}^{e}$ siècle, Paris, 2000, p. 337-340.

3. Pour un exposé synthétique de cette question et la bibliographie: E. DESTEFANIS, «La vie quotidienne des moines et des moniales en Italie du Nord jusqu'au $\mathrm{X}^{\mathrm{e}}$ siècle : état des sources archéologique ", in O. DELOUIS et M. MOSSAKOWSKA-GAUBERT (éd.), La vie quotidienne des moines en Orient et en Occident (IVe- $X^{e}$ siècle), t. 1, Le Caire, 2015, p. 387, n. 3.

4. Communication de J. Raaijmakers «Le réseau de Fulda et la création d'un paysage sacré $\left(2{ }^{\mathrm{e}}\right.$ moitié du $9^{\mathrm{e}}$ siècle) " à la table-ronde : Topographie, circulations et hiérarchie au sein des ensembles monastiques dans l'Occident médiéval, sous la direction de Michel Lauwers, Université de Nice, 18-19 avril 2008.

5. A. CHAVARRIA ARNAU, «Monasterios, campesinos y villae en la Hispania visigoda: la tragica historia del abad Nancto ", in Mélanges d'Antiquité tardive. Studiola in honorem Noël Duval, Turnhout, 2004, p. 113-125.

6. N. REVEYRON, « Forma monasterii. Essai sur l'organisation de l'espace monastique comme mise en forme de l'identité ecclésiologique », Hortus artium medievalium, 20/2 (2014), p. 439-447. 
7. R. AlCiATI, «And the villa became a monastery: Sulpicius Severus' community of Primuliacum ", in H. DEY et E. FENTRESS (éd.), Western monasticism Ante litteram. The space of monastic and the early middle ages, Turnhout, 2011, p. 85-98.

8. G. MOYSE, Les origines du monachisme dans le diocèse de Besançon ( $V^{e}-X^{e}$ siècle), Paris, 1973 (extraits de la BEC, t. 131), p. 147.

INDEX

Index géographique : France/Baume-les-Messieurs

Mots-clés : villae, cellae, cellula, locus, monasterium, abbatiola

\section{AUTEUR}

\section{SÉBASTIEN BULLY}

Chargé de recherche CNRS, UMR ArTeHiS 